\title{
U(1) staggered Dirac operator and random matrix theory*
}

\author{
Bernd A. Berg ${ }^{\mathrm{a}}$, Harald Markum ${ }^{\mathrm{b}}$, Rainer Pullirsch ${ }^{\mathrm{b}}$, and Tilo Wettig ${ }^{\mathrm{c}}$ \\ a Department of Physics, The Florida State University, Tallahassee, FL 32306, USA and Supercomputer \\ Computations Research Institute, The Florida State University, Tallahassee, FL 32306, USA \\ bInstitut für Kernphysik, Technische Universität Wien, A-1040 Vienna, Austria \\ c'Institut für Theoretische Physik, Technische Universität München, D-85747 Garching, Germany
}

\begin{abstract}
We investigate the spectrum of the staggered Dirac operator in 4d quenched U(1) lattice gauge theory and its relationship to random matrix theory. In the confined as well as in the Coulomb phase the nearest-neighbor spacing distribution of the unfolded eigenvalues is well described by the chiral unitary ensemble. The same is true for the distribution of the smallest eigenvalue and the microscopic spectral density in the confined phase. The physical origin of the chiral condensate in this phase deserves further study.
\end{abstract}

By now it is a well-known fact that the spectrum of the QCD Dirac operator

$i D+i m=\left(\begin{array}{cc}i m & T \\ T^{\dagger} & i m\end{array}\right)$ in a chiral basis

is related to universality classes of random matrix theory (RMT), i.e. determined by the global symmetries of the QCD partition function [1]. In RMT the matrix $T$ in Eq. (11) is replaced by a random matrix with appropriate symmetries, generating the chiral orthogonal (chOE), unitary (chUE), and symplectic (chSE) ensemble, respectively [2. For $\mathrm{SU}(2)$ and $\mathrm{SU}(3)$ gauge groups numerous results exist confirming the expected relations [3].

We have investigated $4 \mathrm{~d} \mathrm{U}(1)$ gauge theory described by the action $S\left\{U_{l}\right\}=\sum_{p}\left(1-\cos \theta_{p}\right)$ with $U_{l}=U_{x, \mu}=\exp \left(i \theta_{x, \mu}\right)$ and $\theta_{p}=\theta_{x, \mu}+\theta_{x+\hat{\mu}, \nu}-$ $\theta_{x+\hat{\nu}, \mu}-\theta_{x, \nu} \quad(\nu \neq \mu)$. At $\beta_{c} \approx 1.01 \mathrm{U}(1)$ gauge theory undergoes a phase transition between a confinement phase with mass gap and monopole excitations for $\beta<\beta_{c}$ and the Coulomb phase which exhibits a massless photon $\llbracket$ for $\beta>\beta_{c}$. The question of the order of this phase transition, and hence the issue of a continuum limit $\beta \rightarrow \beta_{c}-0$ of the massive phase, has remained a subject of endless debate, see for instance [5] and

\footnotetext{
*Partially funded by the Department of Energy under contracts DE-FG02-97ER41022 and DE-FG05-85ER2500.
}

references therein. For $\beta>\beta_{c}$ a critical line of continuum theories ought to exist as the photon is massless for all these $\beta$-values.

We are interested in the relationship between $\mathrm{U}(1)$ gauge theory and RMT across this phase transition [6]. The Bohigas-Giannoni-Schmit [7] conjecture states that quantum systems whose classical counterparts are chaotic have spectral fluctuation properties (measured, e.g., by the nearest-neighbor spacing distribution $P(s)$ of unfolded eigenvalues) given by RMT, whereas systems whose classical counterparts are integrable obey a Poisson distribution, $P(s)=\exp (-s)$. As the Dirac operator is a quantum-mechanical object without classical counterpart, the meaning of the Bohigas-Giannoni-Schmit conjecture for lattice gauge theory is somewhat unclear. Nevertheless, as for $\mathrm{SU}(2)$ and $\mathrm{SU}(3)$ gauge groups, we expect the confined phase to be described by RMT, whereas free fermions are known to yield the Poisson distribution. The question arose whether the Coulomb phase will be described by RMT or by the Poisson distribution, with apparently no clear theoretical arguments in favor of either scenario.

In Ref. [6] some of the authors have resolved this question by numerical analysis. We generated twenty (or more) gauge configurations at each of the following parameter values: $8^{3} \times 4$ lattice at $\beta=0,0.9,0.95,1,1.05,1.1,1.5$ and 

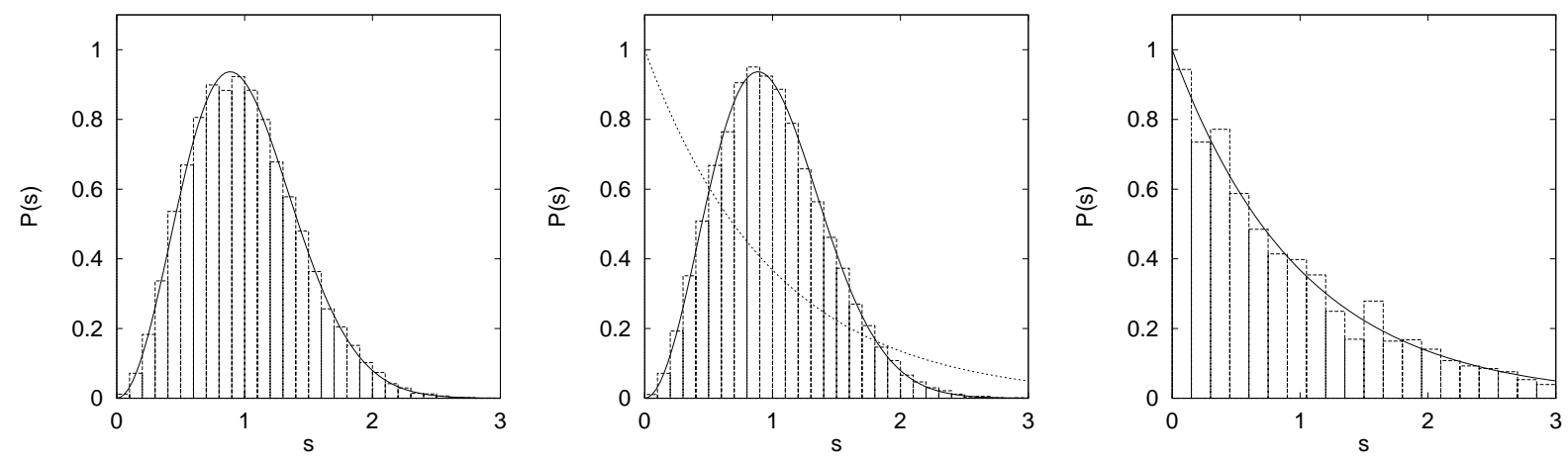

Figure 1. Nearest neighbor spacing distribution $P(s)$ on an $8^{3} \times 6$ lattice in the confined phase (left plot) and in the Coulomb phase (central plot), and for the free Dirac operator on a $53 \times 47 \times 43 \times 41$ lattice (right plot). The theoretical curves are the chUE result, $P(s)=32(s / \pi)^{2} \exp \left(-4 s^{2} / \pi\right)$, and the Poisson distribution, $P(s)=\exp (-s)$.

$8^{3} \times 6$ lattice at $\beta=0.9,1.1,1.5$. The nearestneighbor spacing distributions for the $8^{3} \times 6$ lattice at $\beta=0.9$ (confined phase) and at $\beta=1.1$ (Coulomb phase), averaged over 20 independent configuration, are depicted in Fig. 1. Both are well described by the chUE. In contrast, the right plot in Fig. 11 shows that free fermions are described by the Poisson distribution. The large prime numbers for the lattice size are needed to remove the degeneracies of the spectrum.

We have continued the above investigation with a study of the distribution of small eigenvalues in the confined phase. The Banks-Casher formula [8] relates the eigenvalue density $\rho(\lambda)$ at $\lambda=0$ to the chiral condensate,

$\Sigma=|\langle\bar{\psi} \psi\rangle|=\lim _{m \rightarrow 0} \lim _{V \rightarrow \infty} \pi \rho(0) / V$.

The microscopic spectral density

$\rho_{s}(z)=\lim _{V \rightarrow \infty} \frac{1}{V \Sigma} \rho\left(\frac{z}{V \Sigma}\right)$

should be given by the result for the chUE of RMT [1]. This function also generates the Leutwyler-Smilga sum rules [9].

To study the smallest eigenvalues, spectral averaging is not possible, and one has to produce large numbers of configurations. Our present results are for $\beta=0.9$ in the confined phase with 10000 configurations on a $4^{4}, 10000$ configuration on a $6^{4}$, and 1106 configurations on an $8^{3} \times 6$ lattice. The upper plot in Fig. 2 exhibits the distribution $P\left(\lambda_{\min }\right)$ of the smallest eigenvalue $\lambda_{\min }$ in comparison with the prediction of the (quenched) chUE of RMT for topological charge $\nu=0$,

$$
P\left(\lambda_{\min }\right)=\frac{(V \Sigma)^{2} \lambda_{\min }}{2} \exp \left(-\frac{\left(V \Sigma \lambda_{\min }\right)^{2}}{4}\right) \text {. }
$$

The agreement is excellent for all lattices. For the chiral condensate we obtain $\Sigma \approx 0.35$ by extrapolating the histogram for $\rho(\lambda)$ to $\lambda=0$ and using Eq. (2). (With staggered fermions on a finite lattice one always has $\rho(0)=0$, but within reasonable limits the extrapolated value is independent of the choice of the bin size.) Since the average value of $\lambda_{\min }$ goes like $V^{-1},\left\langle\lambda_{\min }\right\rangle$ decreases with increasing lattice size. In the lower plot of Fig. 2 the same comparison with RMT is done for the microscopic spectral density (3) up to $z=10$, and the agreement is again quite satisfactory. Here, the analytical RMT result for the (quenched) chUE and $\nu=0$ is given by

$\rho_{s}(z)=\frac{z}{2}\left[J_{0}^{2}(z)+J_{1}^{2}(z)\right]$.

The quasi-zero modes which are responsible for the chiral condensate $\Sigma \approx 0.35$ build up when we cross from the Coulomb into the confined phase. For our $8^{3} \times 6$ lattice, Fig. 3 compares on identical scales densities of the small eigenvalues at $\beta=0.9$ (left plot) and at $\beta=1.1$ (right plot), averaged over 20 configurations. The quasi-zero modes in the left plot are responsible for the non-zero chiral condensate $\Sigma>0$ via Eq. (2), whereas no such quasi-zero modes are found in the Coulomb 

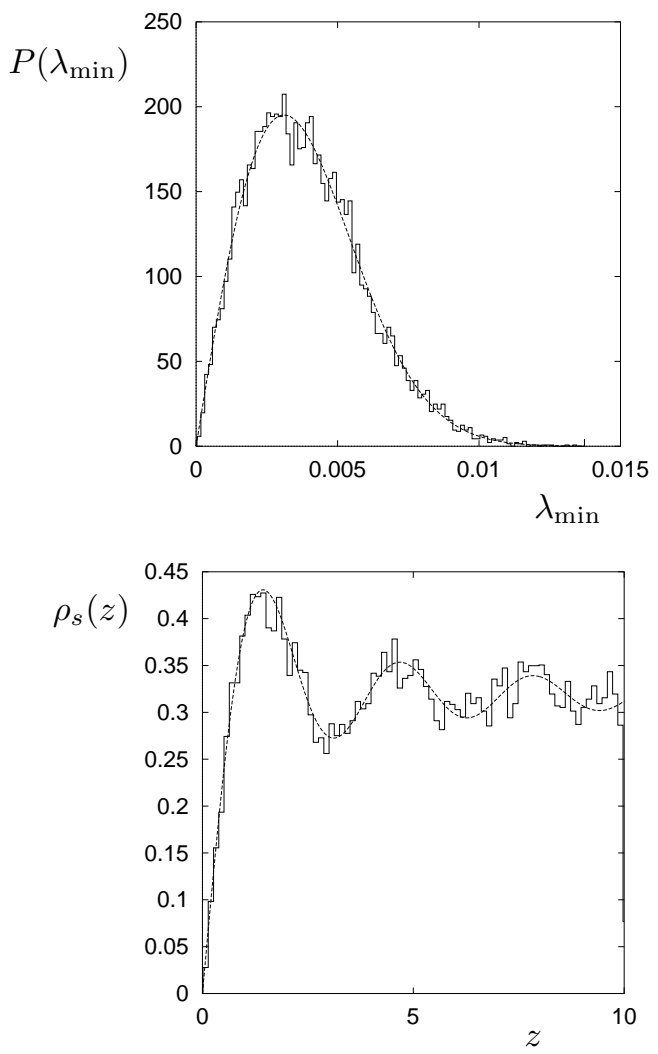

Figure 2. Distribution $P\left(\lambda_{\min }\right)$ (upper plot) and microscopic spectral density $\rho_{s}(z)$ (lower plot) from our $6^{4}$ lattice data in comparison with the predictions of the chUE of RMT (dashed lines), see Eqs. (14) and (5).

phase. This is as expected. However, it may be worthwhile to understand the physical origin of the U(1) quasi-zero modes in more detail. For $4 \mathrm{~d} \mathrm{SU}(2)$ and $\mathrm{SU}(3)$ gauge theories a general interpretation is to link them, and hence the chiral condensate, to the existence of instantons. As there are no instantons in $4 \mathrm{~d} \mathrm{U}(1)$ gauge theory, one needs another explanation, and it may be interesting to study similarities and differences to the $4 \mathrm{~d} \mathrm{SU}(2)$ and $\mathrm{SU}(3)$ situations. An analogous case exists in 3d QCD [10].

In conclusion, the nearest-neighbor spacing distribution of $4 \mathrm{~d} \mathrm{U}(1)$ quenched lattice gauge theory is described by the chUE in both the confinement and the Coulomb phase. In the confinement phase we also find that the $P\left(\lambda_{\min }\right)$ distribution
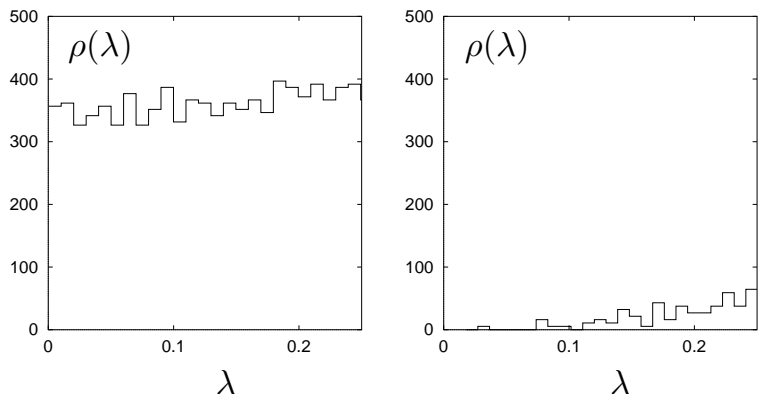

Figure 3. Density $\rho(\lambda)$ of small eigenvalues for the $8^{3} \times 6$ lattice at $\beta=0.9$ (left plot) and at $\beta=1.1$ (right plot). A non-zero chiral condensate is supported in the confinement phase.

and the microscopic spectral density (3) are described by the chUE. A better physical understanding of the origin of the quasi-zero modes, which are responsible for the non-zero chiral condensate, is desirable.

\section{REFERENCES}

1. E.V. Shuryak and J.J.M. Verbaarschot, Nucl. Phys. A560 (1992) 306; J.J.M. Verbaarschot and I. Zahed, Phys. Rev. Lett. 70 (1993) 3852; J.C. Osborn, D. Toublan, and J.J.M. Verbaarschot, Nucl. Phys. B540 (1999) 317; P.H. Damgaard, J.C. Osborn, D. Toublan, and J.J.M. Verbaarschot, Nucl. Phys. B547 (1999) 305.

2. J.J.M. Verbaarschot, Phys. Rev. Lett. 72 (1994) 2531.

3. see, e.g., T. Wettig, hep-lat/9905020.

4. B.A. Berg and C. Panagiotakopoulos, Phys. Rev. Lett. 52 (1984) 94.

5. J. Jersák, C.B. Lang, and T. Neuhaus, Phys. Rev. Lett. 77 (1996) 1933.

6. B.A. Berg, H. Markum, and R. Pullirsch, Phys. Rev. D59 (1999) 097504.

7. O. Bohigas, M.-J. Giannoni, and C. Schmit, Phys. Rev. Lett. 52 (1984) 1.

8. T. Banks and A. Casher, Nucl. Phys. B169 (1980) 103.

9. H. Leutwyler and A.V. Smilga, Phys. Rev. D46 (1992) 5607.

10. J.J.M. Verbaarschot and I. Zahed, Phys. Rev. Lett. 73 (1994) 2288. 